\title{
Post-acute care for stroke - a retrospective cohort study in Taiwan
}

\author{
This article was published in the following Dove Press journal: \\ Patient Preference and Adherence \\ I August 2017 \\ Number of times this article has been viewed
}

\author{
Chung-Liang Lai ${ }^{1, *}$ \\ Ming-Miau Tsai,** \\ Jia-Yuan Luo' \\ Wan-Chun Liao' \\ $\mathrm{Pi}-\mathrm{Shan} \mathrm{Hsu}{ }^{2,3}$ \\ Han-Yu Chen ${ }^{4}$ \\ 'Department of Physical Medicine and \\ Rehabilitation, ${ }^{2}$ Department of Family \\ Medicine, Taichung Hospital, Ministry \\ of Health and Welfare, ${ }^{3} \mathrm{Graduate}$ \\ Institute of Microbiology and \\ Public Health, College of Veterinary \\ Medicine, National Chung-Hsing \\ University, ${ }^{4}$ Department of Physical \\ Therapy, Hungkuang University, \\ Taichung, Taiwan \\ *These authors contributed equally \\ to this work
}

Background: Stroke often causes functional decline in patients. Therefore, after the acute phase, many patients require post-acute care (PAC) to maximize their functional progress, reduce disability, and make it possible for them to return to their home and community. PAC can be provided in different settings. Taiwan's National Health Insurance (NHI) proposed a PAC pilot program, effective since 2014, for stroke patients that allowed patients with the potential for functional improvement to receive PAC rehabilitation in regional or community hospitals. The purpose of this study was to explore the initial achievements and clinical impact of this program in Taiwan.

Methods: This was a retrospective cohort study that mainly analyzed basic hospitalization data and scores for function and quality of life, as recorded immediately after admission and before discharge, for stroke patients in the PAC program in a hospital in Taiwan.

Results: This study collected complete data from a total of 168 patients. After an average of 43.57 days in the program, patients showed significant improvement in the Modified Rankin Scale (MRS), the Barthel Activity Daily Living Index (B-ADL), the Lawton-Brody Instrumental Activity Daily Living Scale (LB-IADL), the Functional Oral Intake Scale (FOIS), and the Mini Nutrition Assessment (MNA), in mobility, self-care, and usual activity, as well as on anxiety/ depression in the EuroQol Five Dimensions Questionnaire (EQ-5D) and in the Mini Mental State Examination (MMSE). After discharge, $76.8 \%$ of the patients could return to their home and community.

Conclusion: This study showed that the pilot PAC program significantly promoted recovery of function in stroke patients and helped them to return to their home and community. Patients with the potential for functional recovery should consider receiving PAC service in a hospital after discharge from acute stroke care.

Keywords: post-acute care, rehabilitation, stroke

\section{Introduction}

In various countries around the world, stroke is a common cause of disability, ${ }^{1}$ and ranks as third in the top ten leading causes of death in Taiwan. Stroke affects nearly 800,000 people in the USA every year ${ }^{2}$ and approximately 40,000 people in Taiwan. Stroke can cause numerous sequelae including limb weakness, sensory deficits, aphasia, dysphagia, hemineglect, visual field deficit, coordination problems, and cognitive impairment. ${ }^{3,4}$ Furthermore, anxiety, depression, and lower physical activity are common symptoms in stroke survivors. ${ }^{5,6}$ Rehabilitative services are the primary mechanism to promote functional recovery and achievement of independence in stroke patients. ${ }^{7}$ After the acute stroke stage, when neurological symptoms and vital signs are stable, patients with functional impairment enter the post-acute stage and undergo rehabilitation. More than two-thirds of stroke survivors receive rehabilitative care. ${ }^{2}$
Correspondence: Han-Yu Chen Department of Physical Therapy, Hungkuang University, No 1018 , Section 6, Taiwan Boulevard, Shalu, Taichung 43302, Taiwan Tel +8864263। 8652 (ext 33।3) Email hychen99@hk.edu.tw 
Patients who still suffer from functional deficits cannot directly return to their home and community after their medical treatment. Therefore, a treatment model as a transitional phase between discharge and returning home is required. At present, there are two systems: the first includes subacute care and post-acute care (PAC) in the USA and the second involves intermediate care in the UK. ${ }^{8-10}$ These care models all aim to achieve improvement in function and successful return to home at a lower cost of hospitalization., ${ }^{2,7}$

PAC rehabilitation for stroke in the USA is highly heterogeneous, varying in the type of care setting, duration, intensity, and the degree of involvement of the physician, nurse, therapist, and other specialists. ${ }^{7}$ With respect to the intensity of rehabilitative care, depending on the setting, the highest intensive rehabilitation care is provided in inpatient rehabilitation facilities (IRFs), followed by skilled nursing facilities (SNFs) which provide subacute nursing and rehabilitation. Long-term care hospitals (LTCHs) are another inpatient setting for PAC rehabilitation. Stroke rehabilitation care can also be provided by a home healthcare agency (HHCA) or at outpatient clinics in the community. ${ }^{7,8}$ There is strong evidence that multidisciplinary and organized inpatient stroke rehabilitation not only reduces mortality rates and the likelihood of institutional long-term care but also enhances functional recovery and increased independence in daily living. ${ }^{11,12}$

Since the beginning of March 1995, Taiwan's universal National Health Insurance (NHI) has covered more than $96 \%$ of Taiwan's population. According to studies from the NHI database, stroke patients have a high readmission rate and prolonged hospital stay. ${ }^{13,14}$ No PAC program existed in Taiwan; therefore, in 2014, the NHI implemented a pilot post-acute care program, named Post-acute Care Cerebrovascular Diseases (PAC-CVD), for stroke. Patients enrolled in this program were referred from acute wards and diagnosed as acute stroke patients in the post-acute phase with the potential for improvement and the intention to receive rehabilitative care. The PAC program was provided in community or regional hospitals. In order to implement a vertically integrated medical system, patients would have to receive an initial evaluation at a medical center and then be referred to a regional or community hospital for this program. It was hoped that this integrated and continuous care model could help patients regain function or reduce their disability. This study analyzed the scores for function and quality of life both before and after this PAC program, and hypothesized that patients would have better function and quality of life after this program. The study discussed the program's clinical impact by comparing it with PAC models in other countries.

\section{Methods}

\section{Subjects}

This study analyzed the de-identified database of patients who met the inclusion criteria of the PAC-CVD program from January 2014 to December 2015 in one regional hospital, which had the most case numbers in this pilot program in central Taiwan. The inclusion criteria of this program were applicable to patients within 1 month of stroke attack (ICD9:430 437), in stable medical condition, with functional status defined as a Modified Rankin Scale (MRS) ${ }^{15}$ score of 2-4, and with the potential for functional recovery after rehabilitation (possessing basic cognition, learning ability, and intention; having sufficient physical strength to maintain a sitting posture with the support of a wheelchair or bed edge for at least 1 hour; and having the ability to proactively participate in the rehabilitation program). The program's closing (discharge) regulations were that the patients showed 1) functional improvement and the ability to receive rehabilitation in the community; 2) no functional improvement based on two continuous functional evaluations; 3) no potential for functional recovery based on care-team evaluation; 4) patients had stayed more than 12 weeks; 5) patients quit this program; or 6) patients died. All patients enrolled in the PAC program signed an informed consent prior to inclusion. This informed consent gave permission to use anonymized medical data for research. This study was approved by the Institutional Review Board of Tasotun Psychiatric Center (Nan-Tou, Taiwan).

\section{Study design}

This retrospective cohort study collected the scores for various scales at admission/enrollment evaluation and discharge closure evaluation to determine their overall functional progress. This study used fall, mortality, readmission, and tube (nasogastric tube, Foley tube, tracheostomy tube) removal rates to determine the care quality of the post-acute care. Overall, patients received follow-up from admission to 14 days after discharge.

According to the NHI's rules for the program, all patients were to receive comprehensive post-acute care in a hospital by a care team composed of a case manager, physiatrist or physician familiar with stroke care, physical therapist, occupational therapist, speech therapist, nutritionist, pharmacist, and social worker. In addition, the care team provided a 1 - to 3-hour intensive rehabilitation program, depending on the 
patient's ability, every weekday. They also convened periodic weekly meetings to report the initial, interim, and closure evaluations of patients, as well as to discuss the goal, plan of treatment, and effectiveness of rehabilitation. During hospitalization, records included basic personal information such as, gender, date of birth, type and site of the stroke, length of stay, whether there was an indwelling tube (tracheostomy, nasogastric, or Foley tubes), and whether these tubes were removed, etc. In addition, assessment of function and quality of life, including the MRS for evaluation of general condition (scale of scores: $0-6$; lower $=$ better performance) ${ }^{15}$ the Barthel Activity Daily Living Index (B-ADL; range of scores: $0-100$; higher $=$ better performance),${ }^{16}$ the Lawton-Brody Instrumental Activity Daily Living scale (LB-IADL; scale of scores: $0-8$; higher $=$ better performance) ${ }^{17}$ the Functional Oral Intake Scale (FOIS; scale of scores: $1-7$; higher $=$ better performance),${ }^{18}$ the Mini Nutrition Assessment (MNA; range of scores: $0-30$; higher $=$ better performance $),{ }^{19}$ the EuroQol Five Dimensions Questionnaire (EQ-5D) for evaluation of quality of life (scale of scores: $1-3$; lower = better performance), ${ }^{20}$ the Berg Balance Scale (BBS) for evaluation of balance function (range of scores: $0-56$; higher $=$ better performance) ${ }^{21}$ the Mini Mental State Examination (MMSE; range of scores: $0-30$; higher $=$ better performance, age and education relative), ${ }^{22}$ and Concise Chinese Aphasia Test $(\text { CCAT; scale of scores: } 1-12 \text {; higher }=\text { better performance })^{23}$ were performed by members of a care team at admission, every 3 weeks, and at discharge (Figure 1).

\section{Statistical analysis}

This study used SPSS WIN version 18 (SPSS, Version 18.0; Chicago, USA) to analyze the research data. Descriptive statistics included the percentage, mean, and standard deviation. This study used the paired $t$-test, which was adjusted by age and neurological severity (MRS), to compare the functional status and quality of life before and after PAC; $P<0.05$ was defined as the level of statistical significance in the two-tailed analysis.

\section{Results}

A total of 169 patients, who were mostly referred from the acute ward of two medical centers, were admitted to and participated in this program at one regional hospital. One patient died due to an acute cardiac event during hospitalization. Therefore, 168 patients completed all evaluations.

As shown in Table 1, the basic information on the 168 patients was as follows: the average age was $63.01 \pm 13.24$ years, including 99 male patients (58.9\%) and 69 female patients (41.1\%); the average number of days after stroke at admission was 15.56 \pm 7.58 ; the average length of stay in the PAC hospital was $43.57 \pm 26.28$ days; 126 patients $(75.0 \%)$ suffered from ischemic stroke and 42 patients $(25.0 \%)$ suffered from hemorrhagic stroke; and 77 patients $(45.8 \%)$ suffered from right hemiplegia, 88 patients (52.4\%) suffered from left hemiplegia, and three patients (1.8\%) suffered from bilateral hemiplegia.

There were 32 patients (19.0\%) with a nasogastric tube, nine patients (5.4\%) with a Foley catheter, and none of the

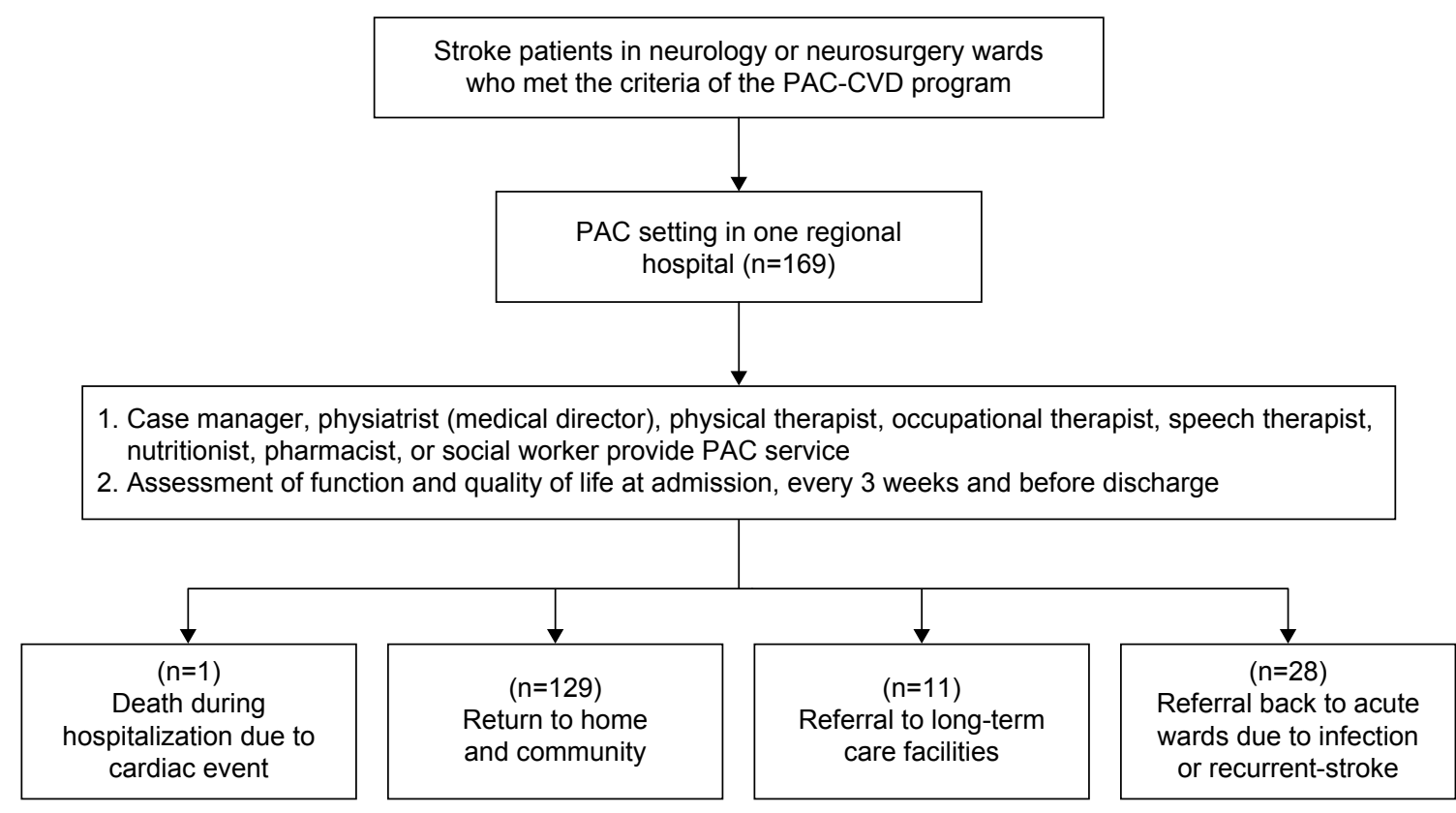

Figure I Flow diagram of healthcare pathway.

Abbreviations: PAC, post-acute care; PAC-CVD, post-acute Care Cerebrovascular Diseases. 
Table I Baseline data of stroke patients

\begin{tabular}{llll}
\hline & N & $\%$ & Mean \pm SD \\
\hline Gender & 99 & 58.9 & \\
$\quad$ Male & 69 & 41.1 & \\
$\quad$ Female & 168 & & $63.01 \pm 13.24$ \\
Age (years) & 88 & 52.4 & \\
$\quad<65$ & 43 & 25.6 & \\
$65-74$ & 37 & 22.0 & \\
$\geq 75$ & & & \\
Affected side & 77 & 45.8 & \\
Left & 88 & 52.4 & \\
$\quad$ Right & 3 & 1.8 & \\
$\quad$ Bilateral & 168 & & $15.56 \pm 7.58$ \\
Days after stroke & & & \\
Stroke type & 126 & 75.0 & $14.33 \pm 7.41$ \\
$\quad$ Ischemic & 42 & 25.0 & $19.02 \pm 7.07$ \\
$\quad$ Hemorrhagic & 168 & & $43.57 \pm 26.28$ \\
Length of stay in the PAC & & & \\
\hline Ab &
\end{tabular}

Abbreviation: PAC, post-acute care.

patients had a tracheostomy tube at admission. The removal rate for the nasogastric tube and Foley catheter was $62.5 \%$ and $88.8 \%$, respectively, after the rehabilitation program. Twenty-five patients $(14.9 \%)$ suffered from a fall, and the mortality rate was $0.6 \%$ (one patient died of a cardiac event) during hospitalization. After discharge, 129 patients (76.8\%) returned to their home and community, 11 patients $(6.5 \%)$ were referred to a long-term care facility, and 28 patients $(16.7 \%)$ were transferred to an acute ward due to infection (urinary traction infection and pneumonia) or recurrent stroke. The readmission rate within 14 days was $5.6 \%$.

On comparing the evaluation after this program against the initial values, there was significant improvement in the MRS, B-ADL, LB-IADL, FOIS, MNA, EQ-5D (Mobility, Self-care, Usual Activities, Anxiety/Depression), BBS, and MMSE and no significant improvement in EQ-5D (Pain/ Discomfort) and CCAT (Table 2). At enrollment, three patients (1.8\%) were classified as MRS 2, 42 patients $(25.0 \%)$ were classified as MRS 3, and 123 (73.2\%) patients were classified as MRS 4 (Table 3). Upon case closure, the level of 69 patients $(41.1 \%)$ improved by $\geq 1$. The comparison of the difference in B-ADL before and after hospitalization showed that the scores of 142 patients $(84.5 \%)$ improved. The score of FOIS in 73 patients (43.4\%) improved as did the score of BBS in 142 patients $(84.5 \%)$. Table 4 shows the change of EQ-5D.

\section{Discussion}

The sequelae of stroke often lead to a great healthcare burden. How stroke patients can be transferred after the acute stage
Table 2 Effect of functional performance and quality of life in patients with stroke

\begin{tabular}{lllll}
\hline & $\mathbf{N}$ & Admission & Discharge & P-value \\
\hline MRS & 168 & $3.71 \pm 0.49$ & $3.26 \pm 0.76$ & $0.000^{*}$ \\
B-ADL & 168 & $44.08 \pm 23.38$ & $70.24 \pm 29.41$ & $0.000^{*}$ \\
LB-IADL & 168 & $1.5 \pm 1.42$ & $3.0 \pm 2.34$ & $0.000^{*}$ \\
FOIS & 168 & $5.48 \pm 1.86$ & $6.33 \pm 1.28$ & $0.000^{*}$ \\
MNA & 168 & $10.72 \pm 3.5 \mathrm{I}$ & $12.70 \pm 5.04$ & $0.000^{*}$ \\
EQ-5D:mobility & 168 & $2.11 \pm 0.42$ & $1.71 \pm 0.57$ & $0.000^{*}$ \\
EQ-5D:self-care & 168 & $2.42 \pm 0.56$ & $1.93 \pm 0.69$ & $0.000^{*}$ \\
EQ-5D:usual activities & 168 & $2.33 \pm 0.53$ & $1.89 \pm 0.62$ & $0.000^{*}$ \\
EQ-5D:pain/discomfort & 168 & $1.19 \pm 0.48$ & $1.13 \pm 0.37$ & 0.203 \\
EQ-5D:anxiety/depression & 168 & $1.32 \pm 0.53$ & $1.14 \pm 0.38$ & $0.000^{*}$ \\
BBS & 168 & $18.48 \pm 16.32$ & $35.98 \pm 18.08$ & $0.000^{*}$ \\
MMSE & 161 & $19.05 \pm 9.22$ & $22.16 \pm 9.84$ & $0.00 I^{*}$ \\
CCAT & 58 & $8.74 \pm 3.88$ & $9.25 \pm 3.91$ & 0.823 \\
\hline
\end{tabular}

Notes: $* P<0.05$. Data presented as mean $\pm S D$ unless otherwise indicated. Abbreviations: MRS, Modified Ranking Scale; B-ADL, Barthel Activity Daily Living index; LB-IADL, Lawton-Brody Instrumental Activity Daily Living scale; FOIS, Functional Oral Intake Scale; MNA, Mini Nutrition Assessment; EQ-5D, EuroQol Five Dimensions questionnaire; BBS, Berg Balance Scale; MMSE, Mini Mental State Examination; CCAT, Concise Chinese Aphasia Test.

to a post-acute care unit, have maximum functional recovery, and return to their home and community is an important research topic. The average age of patients in this study was 63.01 years. After the intervention of the PAC program for an average of 43.57 days, patients displayed significant improvement in the aspects of general condition (MRS), activity of daily living (B-ADL), instrumental activity of daily living (LB-IADL), nutritional status (MNA), quality of life associated with mobility, self-care, usual activity, anxiety/depression (EQ-5D), intake condition (FOIS), balance (BBS), and mental status (MMSE). However, quality of life associated with pain/discomfort (EQ-5D) and Speech function (CCAT) displayed no obvious progress. After discharge, $76.8 \%$ of the patients could return to their home and community. Some review articles have also pointed out the same result with post-care rehabilitation for stroke patients, specifically the reduction of death, dependency, and

Table 3 Ratio of Modified Rankin Scale (MRS) at admission

\begin{tabular}{lll}
\hline Disability level & $\mathbf{N}$ & $\%$ \\
\hline 0 (no symptoms) & 0 & 0 \\
I (no significant disability) & 0 & 0 \\
2 (slight disability) & 3 & 1.8 \\
3 (moderate disability) & 42 & 25.0 \\
4 (moderately severe disability) & 123 & 73.2 \\
5 (severe disability) & 0 & 0 \\
\hline
\end{tabular}

Notes: I, Despite symptoms able to perform all usual duties and activities. 2, Unable to perform all previous activities but able to look after own affairs without assistance. 3, Requires some help, but able to walk without assistance. 4, Unable to walk without assistance and unable to attend to own bodily needs without assistance. 5 , Bedridden, incontinent, and requires constant nursing care and attention. 
Table 4 Number and ratio of the change of EQ-5D after the program

\begin{tabular}{llll}
\hline & Better & Unchanged & Worse \\
\hline Mobility & $69(41.0 \%)$ & $94(56.0 \%)$ & $5(3.0 \%)$ \\
Self-care & $79(47.0 \%)$ & $85(50.6 \%)$ & $4(2.4 \%)$ \\
Usual activities & $69(41.1 \%)$ & $96(57.1 \%)$ & $3(1.8 \%)$ \\
Pain/discomfort & $23(13.7 \%)$ & $129(76.8 \%)$ & $16(9.5 \%)$ \\
Anxiety/depression & $39(23.2 \%)$ & $117(69.6 \%)$ & $12(7.2 \%)$ \\
\hline
\end{tabular}

Abbreviation: EQ-5D, EuroQol Five Dimensions questionnaire.

institutionalization..$^{24,25}$ This program also emphasized that stroke patients in an acute unit could be directly transferred to their community to receive rehabilitation resources as long as their condition was suitable for their return to their home and community by evaluation in the medical center a model similar to Early Supported Discharge (ESD) for American patients. ${ }^{26}$ Therefore, the ratios of MRS 2 and 3 are smaller in Table 3.

In the USA, after acute stroke, approximately $70 \%$ of Medicare beneficiaries received PAC in different settings and most of them availed rehabilitation services. ${ }^{7}$ Some studies compared the stroke patients receiving PAC in IRF and SNF. The results demonstrated that IRF patients had a higher rate of return to their community and greater functional recovery. ${ }^{27,28}$ The biggest difference between them was that IRF provided at least 3 hours of rehabilitation and a physician with rehabilitation training visited every weekday. ${ }^{3,29}$ Another review also deemed that hospital-initiated transitional care could improve the outcome of stroke patients. ${ }^{30}$ In Taiwan, stroke patients received rehabilitation service in rehabilitation wards or community rehabilitation facilities after the acute medical stage; the NHI previously provided no PAC setting; therefore, this PAC program was the first PAC hospital program. The hospital from which this study collected samples provided multidisciplinary rehabilitation team service, including a physiatrist visit and 3 hours of rehabilitation therapy every weekday. The results showed that the readmission rate within 14 days was $5.6 \%$, which was better than that reported in previous studies from the Taiwan NHI database. . $^{14,31}$

In Taiwan, the presence of a nasogastric tube and Foley catheter inserted in the body constituted one of the main reasons why patients and their families were apprehensive about returning to their community, and this prolongs the stay in a hospital or nursing institution. Through the coordinated efforts of physiatrists, nurses, therapists, and nutritionists in this program, the removal rate of the nasogastric tube and Foley catheter was $62.5 \%$ and $88.8 \%$, respectively, which directly increased the return rate of patients to their home and community after discharge. Stroke patients were prone to falls. Between $14 \%$ and $65 \%$ of stroke survivors had fallen in the hospital, while $73 \%$ of stroke survivors had fallen within 6 months after returning home. ${ }^{32}$ Moreover, fear of falling was significantly associated with earlier falls and poor physical function. ${ }^{33}$ BBS was often adopted to evaluate the balance function of stroke patients and to predict the probability of a fall. ${ }^{21}$ This study showed that the score of the BBS scale improved significantly after the intervention of the program, but 25 patients (14.9\%) still fell in the hospital. Therefore, related patient and carer education and prevention of falls in PAC were shown to be vital.

This program required that stroke patients be evaluated by a case manager while they were in the acute stroke unit. If they met the criteria for recruitment into the program, they could be immediately transferred to the hospital within this PAC program so as to reduce their time in the acute unit, resolve the acute unit's lack of beds, and reduce wastage of acute care medical resources. In particular, as the NHI in Taiwan began to gradually implement a different payment system, Diagnosis Related Groups (DRG), the length of stay in the acute unit has been further shortened. The average days from the onset of a stroke to the transfer to the PAC ward (equal to the length of stay in an acute unit) was 15.56 days in this study, compared with the data from the USA; that is, the average length of stay of ischemic stroke patients was 4 days. ${ }^{7}$ Although length of stay in an acute unit was still longer in this program, this was mainly because some acute wards also provided acute rehabilitation services, which increased the length of hospitalization, and there was no stroke DRG in Taiwan. Further, the data in this study included ischemic and hemorrhagic stroke patients. In this program, occupational therapists could help with the training on adaptive equipment and provide suggestions for improving the home environment. Some literature also indicated that such services could increase independence in ADLs. ${ }^{34}$ Furthermore, after returning to their community, a high percentage of stroke patients needed to use household and therapy services. ${ }^{35}$ This program also emphasized early discharge planning during hospitalization and referred patients needing such services to appropriate providers.

In terms of the assessment of quality of life (EQ-5D), anxiety/depression was significantly improved after the intervention of this program. Studies on the assessment of stroke patients found that $31 \%$ of patients suffered from depression, while $27 \%$ experienced anxiety. There was also a high co-morbidity of both conditions. ${ }^{5,36} \mathrm{~A}$ large cohort study of stroke patients evaluated over a 5 -year period indicated 
that, 5 years after the onset of stroke, a high percentage of patients were still experiencing depression (33\%) and anxiety (29\%). Based on this and previous studies, during the PAC stage, or even after returning to their community, stroke patients still had a high potential to have mood issues that should be assessed, prevented, and intervened with as early as possible during PAC. ${ }^{5}$

This study conducted a retrospective analysis of the samples collected from only one hospital. The study's number of samples was the highest in central Taiwan, but some studies have pointed out that geographic variations could have influenced the application of PAC $;{ }^{37}$ therefore, the results of this study could not represent the overall benefits of the PAC-CVD program in Taiwan as a whole. In addition, recruitment of a higher percentage of a younger population ( $<65$ years old) for the study could create a better outcome than recruitment from the general elderly population. However, after the comparison between this study and the relative studies on stroke patients from the Taiwan NHI database, the analytical results of characteristic data were similar, indicating that the patient group in this study was partially representative. ${ }^{13,14,38}$ Moreover, this study excluded stroke patients whose MRS was 5, as their functions were poor in the acute stage. However, some of them could still receive low-intensity $\mathrm{PAC}$ rehabilitation to enhance their functions. The pilot PAC care model for stroke had a positive impact; therefore, the NHI in Taiwan will have more PAC care models in the future. Further prospective studies can randomly assign patients to participate in the program in multiple areas in order to further confirm the effectiveness and clinical impact of this PAC program.

\section{Conclusion}

This study concluded that the pilot PAC program significantly promoted functional recovery for stroke patients in Taiwan. It could help them to return to their home or community earlier and reduce institutionalization. Thus, stroke patients with the potential for functional recovery could receive $\mathrm{PAC}$ in regional or community hospitals after discharge from acute wards.

\section{Acknowledgments}

This research was supported by the PAC team of the Taichung Hospital, Ministry of Health and Welfare.

\section{Disclosure}

The authors report no conflicts of interest in this work.

\section{References}

1. Mukherjee D, Patil CG. Epidemiology and the global burden of stroke. World Neurosurg. 2011;76(6 Suppl):S85-S90.

2. Buntin MB, Colla CH, Deb P, Sood N, Escarce JJ. Medicare spending and outcomes after postacute care for stroke and hip fracture. Med Care. 2010;48(9):776-784.

3. Miller EL, Murray L, Richards L, et al; American Heart Association Council on Cardiovascular Nursing and the Stroke Council. Comprehensive overview of nursing and interdisciplinary rehabilitation care of the stroke patient: a scientific statement from the American Heart Association. Stroke. 2010;41(10):2402-2448.

4. Rathore SS, Hinn AR, Cooper LS, Tyroler HA, Rosamond WD. Characterization of incident stroke signs and symptoms: findings from the atherosclerosis risk in communities study. Stroke. 2002;33(11): 2718-2721.

5. Lincoln NB, Brinkmann N, Cunningham S, et al. Anxiety and depression after stroke: a 5 year follow-up. Disabil Rehabil. 2013;35(2): 140-145.

6. Gebruers N, Vanroy C, Truijen S, Engelborghs S, De Deyn PP. Monitoring of physical activity after stroke: a systematic review of accelerometry-based measures. Arch Phys Med Rehabil. 2010;91(2): 288-297.

7. Winstein CJ, Stein J, Arena R, et al; American Heart Association Stroke Council, Council on Cardiovascular and Stroke Nursing, Council on Clinical Cardiology, and Council on Quality of Care and Outcomes Research. Guidelines for adult stroke rehabilitation and recovery: a guideline for healthcare professionals from the American Heart Association/American Stroke Association. Stroke. 2016;47(6): e98-e169.

8. Buntin MB. Access to postacute rehabilitation. Arch Phys Med Rehabil. 2007;88(11):1488-1493.

9. Woodford HJ, George J. Intermediate care for older people in the U.K. Clin Med (Lond). 2010;10(2):119-123.

10. Melis RJ, Olde Rikkert MG, Parker SG, van Eijken MI. What is intermediate care? BMJ. 2004;329(7462):360-361

11. Prvu Bettger JA, Stineman MG. Effectiveness of multidisciplinary rehabilitation services in postacute care: state-of-the-science. A review. Arch Phys Med Rehabil. 2007;88(11):1526-1534.

12. Stroke Unit Trialists' Collaboration. Organised inpatient (stroke unit) care for stroke. Cochrane Database Syst Rev. 2007;(4):CD000197.

13. Lee HC, Chang KC, Lan CF, Hong CT, Huang YC, Chang ML. Factors associated with prolonged hospital stay for acute stroke in Taiwan. Acta Neurol Taiwan. 2008;17(1):17-25.

14. Tseng MC, Lin HJ. Readmission after hospitalization for stroke in Taiwan: results from a national sample. J Neurolo Sci. 2009;284(1-2):52-55.

15. van Swieten JC, Koudstaal PJ, Visser MC, Schouten HJ, van Gijn J. Interobserver agreement for the assessment of handicap in stroke patients. Stroke. 1988;19(5):604-607.

16. Mahoney FI, Barthel DW. Functional evaluation: the Barthel index. Md State Med J. 1965;14:61-65.

17. Lawton MP, Brody EM. Assessment of older people: self-maintaining and instrumental activities of daily living. Gerontologist. 1969;9(3): 179-186.

18. Crary MA, Mann GD, Groher ME. Initial psychometric assessment of a functional oral intake scale for dysphagia in stroke patients. Arch Phys Med Rehabil. 2005;86(8):1516-1520.

19. Rubenstein LZ, Harker JO, Salvà A, Guigoz Y, Vellas B. Screening for undernutrition in geriatric practice: developing the short-form mini-nutritional assessment (MNA-SF). J Gerontol A Biol Sci Med Sci. 2001;56(6):M366-M372.

20. EuroQol Group. EuroQol - a new facility for the measurement of health-related quality of life. Health Policy. 1990;16(3):199-208.

21. Maeda N, Kato J, Shimada T. Predicting the probability for fall incidence in stroke patients using the Berg Balance Scale. J Int Med Res. 2009; 37(3):697-704 
22. Folstein MF, Folstein SE, McHugh PR. "Mini-mental state". A practical method for grading the cognitive state of patients for the clinician. J Psychiatr Res. 1975;12(3):189-198.

23. Chung YM, Lee SE, Chang MH, Hsu TC. The concise Chinese aphasia test and its application. J Speech Lang Hearing Assoc. 1998;13: 119-137.

24. Collaborative systematic review of the randomised trials of organised inpatient (stroke unit) care after stroke. Stroke Unit Trialists' Collaboration. BMJ. 1997;314(7088):1151-1159.

25. Langhorne $P$, Duncan $P$. Does the organization of postacute stroke care really matter? Stroke. 2001;32(1):268-274.

26. Langhorne P, Holmqvist LW; Early Supported Discharge Trialists. Early supported discharge after stroke. J Rehabil Med. 2007;39(2): 103-108.

27. Deutsch A, Granger CV, Heinemann AW, et al. Poststroke rehabilitation: outcomes and reimbursement of inpatient rehabilitation facilities and subacute rehabilitation programs. Stroke. 2006;37(6):1477-1482.

28. Chan L, Sandel ME, Jette AM, et al. Does postacute care site matter? A longitudinal study assessing functional recovery after a stroke. Arch Phys Med Rehabil. 2013;94(4):622-629.

29. Wang H, Camicia M, Terdiman J, Mannava MK, Sidney S, Sandel ME. Daily treatment time and functional gains of stroke patients during inpatient rehabilitation. PM R. 2013;5(2):122-128.

30. Prvu Bettger J, Alexander KP, Dolor RJ, et al. Transitional care after hospitalization for acute stroke or myocardial infarction: a systematic review. Ann Intern Med. 2012;157(6):407-416.
31. Wu KY, Wu SC, Hung YN, Wu CC, Lin LC, Hu HH. The need for post-acute care for stroke patients in Taiwan. Taiwan Gong Gong Wei Sheng Za Zhi. 2012;31(3):251.

32. Batchelor F, Hill K, Mackintosh S, Said C. What works in falls prevention after stroke?: a systematic review and meta-analysis. Stroke. 2010;41(8):1715-1722.

33. Andersson AG, Kamwendo K, Appelros P. Fear of falling in stroke patients: relationship with previous falls and functional characteristics. Int J Rehabil Res. 2008;31(3):261-264.

34. Legg LA, Drummond AE, Langhorne P. Occupational therapy for patients with problems in activities of daily living after stroke. Cochrane Database Syst Rev. 2006;(4):CD003585.

35. White JH, Alston MK, Marquez JL, et al. Community-dwelling stroke survivors: function is not the whole story with quality of life. Arch Phys Med Rehabil. 2007;88(9):1140-1146.

36. Hackett ML, Pickles K. Part I: frequency of depression after stroke: an updated systematic review and meta-analysis of observational studies. Int J Stroke. 2014;9(8):1017-1025.

37. Kane RL, Lin WC, Blewett LA. Geographic variation in the use of post-acute care. Health Serv Res. 2002;37(3):667-682.

38. Chang KC, Tseng MC. Costs of acute care of first-ever ischemic stroke in Taiwan. Stroke. 2003;34(11):e219-e221.
Patient Preference and Adherence

\section{Publish your work in this journal}

Patient Preference and Adherence is an international, peer-reviewed, open access journal that focuses on the growing importance of patient preference and adherence throughout the therapeutic continuum. Patient satisfaction, acceptability, quality of life, compliance, persistence and their role in developing new therapeutic modalities and compounds to optimize

\section{Dovepress}

clinical outcomes for existing disease states are major areas of interest for the journal. This journal has been accepted for indexing on PubMed Central. The manuscript management system is completely online and includes a very quick and fair peer-review system, which is all easy to use. Visit http://www. dovepress.com/testimonials.php to read real quotes from published authors. 